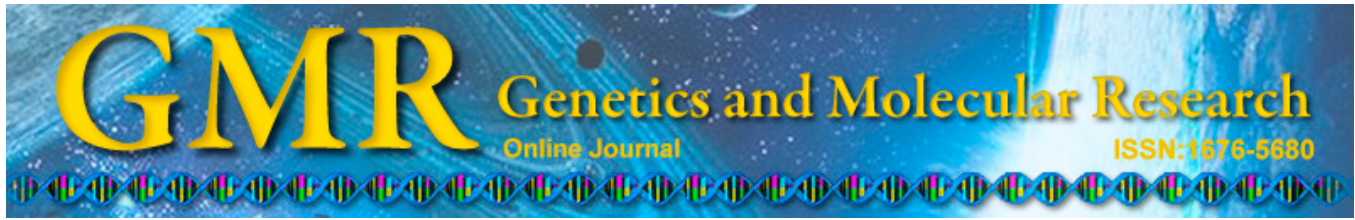

\title{
Computed tomography-guided percutaneous microwave ablation therapy for lung cancer
}

\author{
Y.H. Sun, P.Y. Song, Y. Guo and L.J. Sheng \\ Oncology, Jinan, Shandong Province, China \\ Corresponding author: P.Y. Song \\ E-mail: songyapeng_syp@163.com \\ Genet. Mol. Res. 14 (2): 4858-4864 (2015) \\ Received May 9, 2014 \\ Accepted October 21, 2014 \\ Published May 11, 2015 \\ DOI http://dx.doi.org/10.4238/2015.May.11.18
}

Affiliated Hospital of Shandong Province Academy of Medical Sciences

\begin{abstract}
This study evaluated the clinical efficacy and value of computed tomography (CT)-guided percutaneous microwave ablation therapy (PMAT) for lung cancer without surgical treatment. A total of 39 lesions in 29 patients with peripheral lung cancer were treated by CT-guided PMAT under local anesthesia. The microwave energy was $50-70 \mathrm{~W}$ at a frequency of $2450 \mathrm{MHz}$. The treatment was performed by using 1 or 2 points of ablation emission according to the size and shape of the tumor. Operations were completed in 29 patients. The average operating time was $8 \mathrm{~min}$ (range: $5-12 \mathrm{~min}$ ). After PMAT, lower density in the ablated area was observed by CT. Pre- and posttreatment CT values were 52.60 and $26.12 \mathrm{Hu}$, respectively. Eight, 14,4 , and 3 patients achieved complete remission, partial remission, stable status, and progression, respectively, for an effectiveness rate of $75.86 \%$. Complications included 5, 2, and 15 cases of pneumothorax, pleural effusion, and fever, respectively. No needle track implantation was observed. Mean progression-free survival was 14.6 months. The 1- and 2-year survival rates were 91.3 and $82.6 \%$, respectively. Thus,
\end{abstract}


PMAT is a minimally invasive, safe, and effective treatment for lung cancer. It can improve quality of life, prolong survival, and improve the survival rate.

Key words: CT-guided; Percutaneous microwave ablation therapy; Lung cancer

\section{INTRODUCTION}

Surgical resection is currently the mainstay of treatment for lung cancer. However, only approximately $15 \%$ of patients have clinically resectable cancers (Shu et al., 2011). Local nonsurgical thermal ablation therapies such as microwave ablation are considered important approaches to treat patients who cannot tolerate surgery because of cardiopulmonary dysfunctions or other complications, advanced disease, unwillingness to undergo surgical resection, or non-resectable tumors. Previous studies demonstrate the efficacy of percutaneous microwave ablation therapy (PMAT) for the treatment of lung cancer (Wang et al., 2010). However, relevant research information and data are limited. Therefore, the present study evaluated the efficacy and value of PMAT under computed tomography (CT) guidance in patients with peripheral lung cancer.

\section{MATERIAL AND METHODS}

\section{Demographic data}

Between March 2010 and February 2014, 29 patients diagnosed with primary or metastatic lung cancer by pulmonary biopsy and sputum cytology and admitted to the Affiliated Hospital of the Shandong Academy of Medical Science were treated with PMAT. There were 17 males and 12 females aged between 39 and 74 years with a median age of 63 years. Of the 29 patients, 15 were diagnosed with primary lung cancer, while 14 were diagnosed with metastatic cancers in the lungs, including 8,4 , and 2 cases of metastatic intestinal adenocarcinoma, primary liver cancer metastasis, and metastatic breast cancer, respectively. A total of 39 lesions were identified in these patients: 17 had 1 lesion, while 12 had 2-3 lesions all located on the same side of the lung. The tumor diameters ranged from $1.5-5.8 \mathrm{~cm}$, with a mean diameter of $3.7 \mathrm{~cm} ; 14$ patients had lesions $1.5-3.0 \mathrm{~cm}$, while 15 had lesions $3.1-5.8 \mathrm{~cm}$. None of the patients presented with hemorrhagic tendency or coagulation dysfunction. Among the patients with primary lung cancer, 7, 2, 4, and 2 had stage I, II, III, and IV lung cancer, respectively. Distant metastatic lesions of stage IV primary lung cancers and the primary tumors of the patients with metastatic cancers in lungs all achieved complete response (CR) or stable disease (SD) after undergoing previous treatment such as surgical resection, general chemotherapy, etc.

\section{Methods}

\section{Pre-ablation preparations}

The size, location, and number of tumors were evaluated by conventional CT scan. Routine blood tests, prothrombin time and activated partial thromboplastin time evaluation, liver and kidney function tests, and electrocardiography were all conducted prior to ablation 
therapy. All patients fasted for 8-12 h prior to the therapy and were injected with $10 \mathrm{mg}$ morphine combined with $10 \mathrm{mg}$ diazepam 30 minutes before treatment. Signed written informed consent for microwave ablation therapy was obtained from all patients or their family members prior to treatment.

\section{Therapeutic instruments}

A KY-2000 Microwave Ablation System (Nanjing Kangyou Medical Microwave Institute, China) with a microwave frequency of $2450 \mathrm{MHz}$ and output power of 10-100 W (continuously adjustable) as well as a 64-section spiral CT system (Siemens Healthcare, Germany) were used.

\section{Therapeutic methods}

The patients were placed in the supine or prone position. External electrocardiography monitoring and intravenous access were placed. The entry route of the ablation was determined with reference to pre-ablation CT scans. While avoiding the major blood vessels and peripheral bones (e.g., the ribs, scapula, etc.), the nearest point between the chest skin and the center of the tumor was selected as the puncture point. The microwave applicator was introduced along the same direction as the line connecting the puncture point and tumor center. After the insertion angle and depth were determined, $5 \mathrm{~mL} \mathrm{2 \%} \mathrm{lidocaine} \mathrm{was} \mathrm{injected} \mathrm{into} \mathrm{the}$ puncture point for local anesthesia. The patients were instructed to hold their breath, and the microwave antenna was directly inserted until it reached the predetermined location. CT scanning was performed again to confirm the location of the antenna tip and subsequently connect the antenna with the microwave ablation instrument and water-cooling device. The microwave frequency used was $2450 \mathrm{MHz}$ at $50-70 \mathrm{~W}$. The range of ablation was $0.5-1.0 \mathrm{~cm}$ beyond the tumor margin. Single- and double-point ablation were applied to tumors $\leq 3$ and $>3 \mathrm{~cm}$ in diameter, respectively. When the ablation procedure was completed, the patient was again asked to hold their breath while the therapeutic antenna was removed slowly and gradually. The needle passage was cauterized for hemostasis and to prevent the tumor spreading from the passage. The patient underwent CT scan immediately after ablation; any changes in the lesions as well as any pneumothorax/hemothorax conditions were monitored.

\section{Evaluation of complications and efficacy}

Any associated symptoms occurring within 30 days following ablation were considered therapy-related complications. CT review was conducted 1, 3, and 6 months after therapy to observe the changes in the lesions. Efficacy was evaluated according to the Response Evaluation Criteria In Solid Tumors version 1.1; patients' conditions were classified into the following 4 categories: CR, partial response (PR), $\mathrm{SD}$, and progressive disease (PD); the response rate was determined by combining CR and PR (Fernarido et al., 2005)

\section{Follow-up}

The end of follow-up was February 2014; by this time, the patients had been followed for 3-45 months with a median of 25 months. None of the patients were lost to follow-up ( $100 \%$ attendance). Progression-free survival was defined as the time from study enrollment until the progression of the lung lesions had been treated with ablation. 


\section{RESULTS}

\section{Treatment and efficacy}

Fourteen patients with lesions from $1.5-3.0 \mathrm{~cm}$ were treated with single-point ablation, while 15 patients with lesions from $3.1-5.8 \mathrm{~cm}$ were treated with double-point ablation. PMAT was performed successfully in all patients; the procedure ranged from 5-12 min with a mean of 8 min. Post-ablation CT scans showed low-density opacity in the cancer tissues surrounded by high-density peripheral tissues. The CT measurement decreased from $52.60 \mathrm{Hu}$ pre-ablation to $26.12 \mathrm{Hu}$ post-ablation. Enhanced CT follow-up 1 month post-ablation showed enhancement in 29 lesions and enhancement limited to the peripheral regions in 10 lesions. Cavitations were noted in most lesions. Enhanced CT scans 3 months post-ablation performed to evaluate treatment efficacy showed 8, 14, 4, and 3 patients achieved CR (i.e., no enhancement occurred within the lesion or peripheral tissues, and the lesions also changed to fibrous scars with increasing time), $\mathrm{PR}, \mathrm{SD}$, and PD, respectively. Hence, the response rate was $75.86 \%$.

\section{Follow-up observations}

Twenty-one patients survived until the end of the study. The 1-year local control rate was $65.2 \%$, and the median progression-free survival was 14.6 months. The 1- and 2-year survival rates were 91.3 and $82.6 \%$, respectively.

\section{Complications}

Intra-ablation local pain and burning sensation occurred in 17 patients; all of these symptoms were tolerable, and no post-ablation local discomfort was reported. On immediate post-ablation CT scans, 5 patients presented with pneumothorax; there was 1 case of severe pneumothorax, which was immediately treated with closed drainage of the thoracic cavity and was confirmed to be completely relieved by chest radiography at $48 \mathrm{~h}$. The other 4 patients presented with mild pneumothorax; therefore, no special treatment was administered, and the symptoms relieved spontaneously within 1 week. Meanwhile, 2 patients presented with mild pleural effusion that did not require pleural puncture or drainage; these effusions self-absorbed within 1 month. Furthermore, patients presented with fever within 2-7 days after treatment; their body temperatures ranged from $37.5-38.8^{\circ} \mathrm{C}$, and the fever lasted $3-5$ days. These patients were treated symptomatically with anti-inflammatory or antipyretic treatments and resumed to normal after treatment. No patients died within 1 month after treatment. No patients presented with intrathoracic hemorrhage, needle passage bleeding, or metastasis.

\section{DISCUSSION}

Thermal ablation therapy is a recently developed minimally invasive thermal therapy for the treatment of tumors (Vogl et al., 2011). As a local thermal ablation technique, PMAT has been widely accepted and applied in the treatment of various solid tumors. Furthermore, its importance as a minimally invasive intervention is increasingly being recognized. The heat-insulating effect of the normal air in the lungs surrounding the tumor could facilitate the 
aggregation of heat energy, which enables the thermal ablation of lung tumors (Gadaleta et al., 2004). In contrast to radiofrequency ablation, microwave ablation is not subjected to the influences of electric current or resistance, and carbonization and pneumatolysis of peripheral tissues would not affect the accumulation of heat energy within the tumor tissues, resulting in higher temperature within the tumor tissues. In addition, as the placement of electrode plate is not required, injury to the normal peripheral lung tissues is substantially reduced (Shu et al., 2011). Microwave ablation has several advantages such as rapid temperature increase, higher intra-tumor temperature, shorter treatment duration, direct action, and larger ablation range (Fan and Ye, 2012). During the ablation process, the microwave electric field at $2450 \mathrm{MHz}$ can induce intramolecular friction, which allows a maximum intratumor temperature exceeding $100^{\circ} \mathrm{C}$ and a peripheral temperature up to $60^{\circ} \mathrm{C}$. In addition to achieving rapid necrosis of the tumor cells, ablation can also facilitate the coagulation of peripheral blood vessels, impairing the blood supply to the tumor tissues. The immediate decrease in lesion size on post-treatment CT scan, low-density opacity in the center of the tumor in contrast to the high-density peripheral regions, and the various degrees of shrinkage or disappearance of the lesions following ablation in the present study corroborate all of these advantages.

The clinical efficacy of microwave ablation in the treatment of primary lung cancer and metastatic cancers in the lungs and consequent prognosis are slightly different ( $\mathrm{Lu}$ et al., 2012). In the present study, both PD cases were patients with metastatic cancer in the lungs. $\mathrm{CT}$ review at 3 months post-ablation showed peripheral enhancement and slight lesion enlargement, suggesting tumor relapse. Even in patients whose malignancies were successfully eradicated by PMAT, local tumor progression might still occur.

Single-point microwave ablation complete ablated the lung cancer lesions $<3 \mathrm{~cm}$ in diameter; however, as most tumors $>5 \mathrm{~cm}$ diameter are irregularly shaped, single-point ablation may lead to a residual tumor post-ablation (Nakahara et al., 2005). In the present study, although the tumors $>3 \mathrm{~cm}$ in diameter were treated with double-point ablation, the complete ablation rate was significantly higher in patients with tumors $<3 \mathrm{~cm}$ in diameter than those with tumors $>3 \mathrm{~cm}$ in diameter. Of the $6 \mathrm{CR}$ cases, 5 had tumors $<3 \mathrm{~cm}$ in diameter. This difference is largely attributable to the irregular shapes of larger tumors; even when doublepoint ablation was used, the range of ablation still could not cover the entire lesion. Therefore, double- or multiple-point ablation is recommended for irregularly shaped tumors or those $>5$ $\mathrm{cm}$ in diameter. Guo et al. (2009) suggest that the area of ablation should be at least $0.5-1.0 \mathrm{~cm}$ beyond the margins of the tumor in order to achieve complete tumor necrosis.

By using a needle electrode to emit microwaves within the tumors, microwave ablation therapy takes advantages of the thermal effect of microwaves and the poor heat tolerance of tumors; it increases the local temperature to $100^{\circ} \mathrm{C}$ very rapidly, causing high-temperature carbonization and necrosis of the tumor tissues with only minimal damage to the normal peripheral tissues.

Wolf et al. (2008) retrospectively evaluated 50 patients ( 82 lesions) with metastatic cancers in the lungs ( $>3 \mathrm{~cm}$ in diameter) who underwent CT-guided PMAT and had a mean follow-up of 10 months. After PMAT, 26 and $22 \%$ of the patients presented with residual and relapsed tumors, respectively. The 1 -year control rate was $67 \%$, while the average time to initial recurrence was 16.2 months. After ablation therapy, the 1-, 2-, and 3-year survival rates were 65, 55, and 45\%, respectively. Feng et al. (2002) administered PMAT under CT guidance by using a monopole microwave ablation needle antenna to treat peripheral lung cancers; 28 lesions in 20 patients ( 8 with primary lung cancers, and 12 with metastatic cancers in the 
lungs) were irradiated with a $65-\mathrm{W}$ microwave at $2450 \mathrm{MHz}$ for $60 \mathrm{~s}$. The patients were followed for 3-24 months. Sixteen patients were alive at the end of follow-up. All tumors shrank: 13 lesions exhibited $>50 \%$ shrinkage, and 3 lesions disappeared. Thus, the response rate was $57.1 \%$. In the present study, 23 patients with lung cancer were followed for 3-43 months; 17 survived to the end of follow-up. Furthermore, 6 (26.1\%), $12(52.2 \%), 3(13.0 \%)$, and 2 $(8.7 \%)$ patients achieved CR, PR, SD, and PD, respectively. The 1-year local control rate was $65.2 \%$, and median progression-free survival was 14.6 months. Thus, the present results are generally similar to those studies mentioned above. However, the 1- and 2-year survival rates following ablation are better than those in the previous studies; this is probably because of the relatively smaller proportion of patients with advanced metastatic cancers.

At present, therapeutic efficacy is mainly evaluated by enhanced CT scanning, which could reflect the blood supply to the lesions and peripheral tissues. The absence of enhanced signals within the lesions and peripheral tissues on multiple enhanced scans may suggest the absence of blood supply to the lesion, implying complete tumor necrosis. In the present study, 2 lesions exhibited significant peripheral enhancement and slight enlargement of the masses 3 months after ablation; this is probably attributable to the larger sizes of these tumors and incomplete microwave ablation.

Pneumothorax is the most common complication of microwave treatment, with a reported incidence ranging from 9-52\% (Akboru et al., 2005; Kim et al., 2005); most of the symptoms are caused by puncture by the microwave antennae. In the present study, 3 patients $(13.0 \%)$ presented with pneumothorax, including 1 with severe pneumothorax, which was treated with closed drainage of the thoracic cavity. Chest X-ray conducted $48 \mathrm{~h}$ after drainage confirmed the complete relief of pneumothorax. The other 2 patients had milder pneumothorax, which resolved spontaneously within 1 week. In addition, 2 patients presented with mild pleural effusions that self-absorbed within 1 month without treatment. The occurrence of postablation pleural effusion is mainly associated with local inflammatory exudation of the tumor or peripheral lung tissues after heat exposure (Zhu et al., 2006). In the present study, 15 patients presented with post-ablation fever, which is probably associated with the post-ablation necrotic absorption of tumor tissue as well as the aseptic inflammation caused by localized heating. Microwave ablation allows the coagulation and occlusion of capillaries; therefore, severe pulmonary congestion is rare if precautions are taken to protect major blood vessels. For tumors located at the peripheral regions of the mediastinum, nerves, heart, or major blood vessels, extra precautions should be taken when conducting ablation procedures in order to avoid damage. For lesions that fail to achieve complete necrosis, absolute alcohol can be injected intraoperatively to cause coagulation necrosis of residual tumor cells via dehydration. Hot distilled water can also be used to kill cancer cells, while its lower permeability could also induce the disintegrative necrosis of cancer cells, further resulting in complete ablation. It should also be noted that patients should be asked to hold their breath during both the insertion and removal of the microwave ablation antennae so as to avoid damaging the surface lung tissue. Heating can be performed during the removal of the needles to prevent needle passage bleeding and metastasis.

Notably, microwave ablation is not indicated for patients with central lung cancers or poor lung function. The puncture procedure should be performed carefully to avoid damage and bleeding to major chest blood vessels and larger bronchi, bronchopleural fistula, and severe pneumothorax. There are several considerations that should be taken during the ablation operation. First, the shortest lung puncture pathway should be selected, i.e., the nearest 
chest wall from the lesion should be used as the puncture point to minimize damage to normal lung tissue. Second, the puncture direction should be perpendicular to the pleura in order to reduce the shear force exerted on the pleura. Third, the number of lung punctures should be minimized in order to avoid damage to blood vessels and nerves. Fourth, the puncture should be performed rapidly to reduce damage to the pleura. The patient's vital signs should be monitored closely during the ablation process, alerting the surgeons to any changes in the patient's conditions. The surgeon should confirm the correct insertion of the microwave ablation needles into the intratumor region under $\mathrm{CT}$ guidance. The microwaves should be emitted within a safe range to ensure the efficacy and safety of each ablation operation.

In conclusion, PMAT is a safe, minimally invasive, and efficacious therapy for treating lung cancers. For patients who cannot undergo surgical resection because of cardiorespiratory dysfunction, unwillingness, or advanced metastatic cancers in the lungs, PMAT can effectively deactivate the lesions in situ, reducing the disease burden and improving therapeutic efficacy. Thus, PMAT is a potential nonsurgical therapeutic option with strong implications in the treatment of lung cancers. The combination of PMAT with general interventions (e.g., surgical resection, radiotherapy, chemotherapy, biotherapy, gene therapy, etc.) is expected to reduce tumor cell metastasis as well as improve patient quality of life and survival.

\section{REFERENCES}

Akboru MH, Dincer ST, Yoney A, Askaoglu B, et al. (2005). Concomitant chemo radiotherapy with orally used agents in locally advanced gastric carcinoma: two years results. J. Clin. Oncol. 23: 14175.

Fan WJ and Ye X (2012). Microwave ablation therapy for cancers. People's Medical Publishing House Co., Ltd., Beijing. Fernarido HC, De Hoyos A, Landrenean RJ, Gilbert S, et al. (2005). Radiofrequency ablation for the treatment of nonsmall cell lung cancer in marginal surgical candidates. J. Thorac. Cardiovasc. Surg. 129: 639-644.

Feng W, Liu W, Li C, Li Z, et al. (2002). Percutaneous microwave coagulation therapy for lung cancer. Zhonghua Zhong Liu Za Zhi 24: 388-390.

Gadaleta C, Mattidi V, Colucci G, Cramarossa A, et al. (2004). Radiofrequency ablation of 40 lung neoplasms: preliminary results. AJR Am. J. Roentgenol. 183: 361-368.

Guo CY, Hu HT and Li HL (2009). CT-guided percutaneous microwave coagulation therapy for peripheral lung cancer. Contemp. Med. 15: 674-676.

Kim S, Lim DH, Lee J, Kang WK, et al. (2005). An observational study suggesting clinical benefit for adjuvant postoperative chemoradiation in a population of over 500 cases after gastric resection with D2 nodal dissection for adenocarcinoma of the stomach. Int. J. Radiat. Oncol. Biol. Phys. 63: 1279-1285.

Lu Q, Li XF and Han Y (2012). Analysis of clinical curative effect of microwave ablation in lung cancer. China Medicine 7: 557-559.

Nakahara Y, Mochiduki Y, Miyamoto Y, Nakahara Y, et al. (2005). Prognostic significance of the lymphocyte-toneutrophil ratio in percutaneous fine-needle aspiration biopsy specimens of advanced nonsmall cell lung carcinoma. Cancer 104: 1271-1280.

Shu XL, Shan GL and Ye X (2011). Thermal ablation of lung neoplasms. J. Int. Oncol. 38: 382-385.

Vogl TJ, Naguib NN, Lehnert T and Nour-Eldin NE (2011). Radiofrequency microwave and laser ablation of pulmonary neoplasms: clinical studies and technical consideration - review article. Eur. J. Radiol. 77: 346-357.

Wang Q, Liu RB and Zhang LC (2010). Advances in microwave coagulation therapy of lung cancer. Chin. J. Lung Cancer 13: 78-81.

Wolf FJ, Grand DJ, Machan JT, Dipetrillo TA, et al. (2008). Microwave ablation of lung malignancies: effectiveness, CT findings, and safety in 50 patients. Radiology 247: 871-879.

Zhu LM, Li QS and Lin YX (2006). Percutaneous microwave coagulation therapy for peripheral lung cancer: a short-term evaluation of 16 cases. Chin. J. Minim. Invasive Surg. 6: 115-116. 